\title{
Tuning of disordered local structure in Prussian Blue analogues
}

\section{Arkadiy Simonov, Yevheniia Kholina}

\author{
ETH Zürich
}

arkadiy.simonov@mat.ethz.ch

Disorder is commonly used in chemistry for designing functional materials. For instance, preparation of solid solutions is nothing else than the introduction of a controlled number of point defects in a crystal. Disordered systems, though, provide more degrees of freedom: not only the number of defects, but also their distribution can be used to optimise the functional properties of materials, however up until now, defect distribution was hard to control and thus was rarely used in practice.

In this talk we will show how to precisely tune distribution of point defects by changing various chemical parameters during crystal growth and characterise it with the single crystal diffuse scattering [1].

We will use Prussian Blue Analogues (PBAs) as our model system. PBAs is a class of cyanide materials with the general formula $\mathrm{M}\left[\mathrm{M}^{\prime}(\mathrm{CN})_{6}\right]_{1-\delta} * \mathrm{xH} 2 \mathrm{O}$ where $\mathrm{M}$ and $\mathrm{M}$ ' are transition metals.

Depending on the nature of transition metals, PBAs can accommodate a large number of vacancies on the M'(CN) 6 site (for instance $\delta=0.33$ for $\mathrm{M}=\mathrm{Mn}$ and $\mathrm{M}^{\prime}=\mathrm{Co}$ ) which makes them highly porous and, as a result, attractive for hydrogen storage applications. Distribution of $\mathrm{M}^{\prime}(\mathrm{CN}){ }_{6}$ vacancies is important for the performance of this material, since more disordered vacancy configurations provide more diffusion pathways through the structure, larger accessible volume, and easier transport.

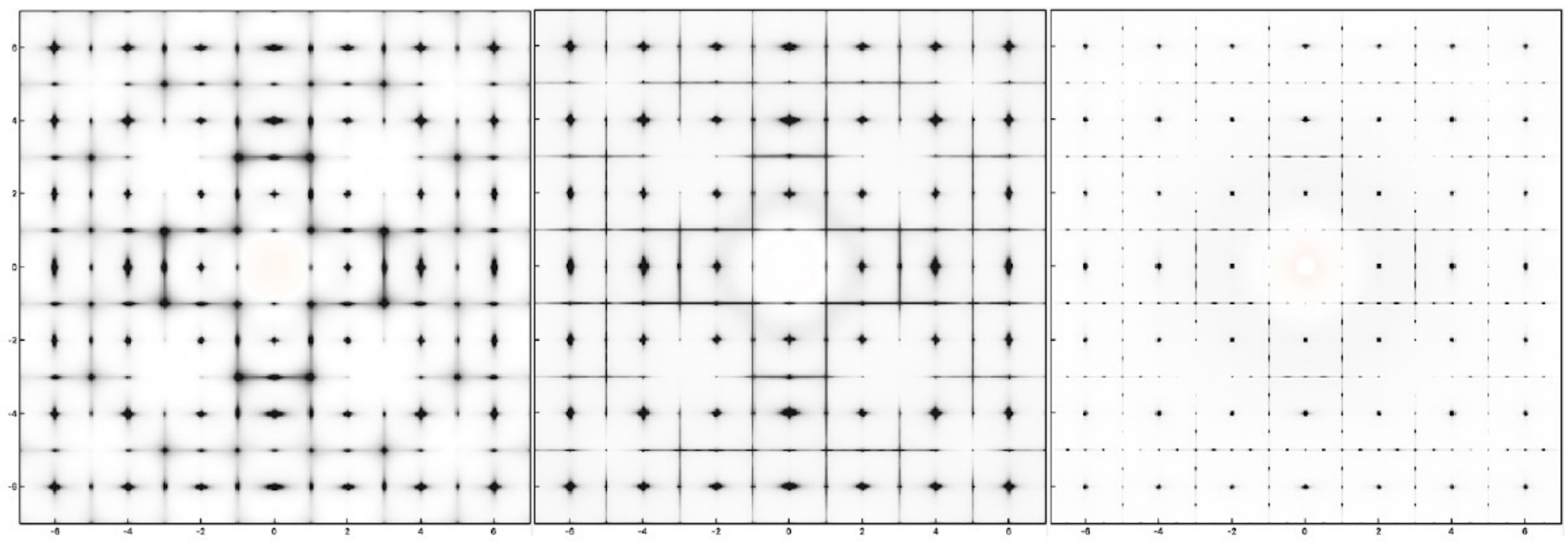

[1] Simonov, Arkadiy, et al. "Hidden diversity of vacancy networks in Prussian blue analogues." Nature 578.7794 (2020): $256-260$.

Keywords: Single crystal diffuse scattering, disorder engineering, local structure, 3D-PDF 